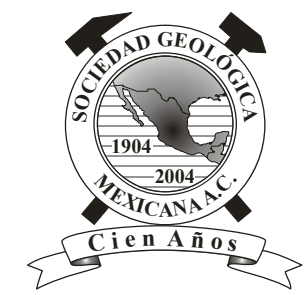

\title{
Diseño y aplicación de instrumentación para la caracterización hidrometeorológica e hidrogeológica
}

\author{
Javier Salas-García ${ }^{1, *}$, Jaime Gárfias ${ }^{1}$, Hilario Llanos², Richard Martel ${ }^{3}$ \\ ${ }^{1}$ Universidad Autónoma del Estado de México, Facultad de Ingeniería. México. Facultad de Ingeniería, Cerro de Coatepec, Ciudad \\ Universitaria, Toluca, Estado de México, C.P.50130. \\ ${ }^{2}$ Universidad del País Vasco. Departamento de Geodinámica. Los Apraiz, 1. Vitoria-Gasteiz, C.P. 01006. País Vasco. \\ ${ }^{3}$ Institut National de la Recherche Scientifique. Centre Eau, Terre \& Environnement. 490 de la Couronne. Québec (Qc). G1K 9A9 \\ Canadá. \\ *proyectos@javiersalasg.com
}

\section{Resumen}

El conocimiento del ciclo hidrológico es un requisito indispensable para la evaluación, la planificación y el desarrollo de los recursos hídricos. En este sentido, el presente artículo describe el principio de funcionamiento, el diseño, los resultados de las pruebas de laboratorio y la instalación en el campo de cinco dispositivos: un pluviómetro, un evaporímetro, un limnígrafo, un freatímetro y un higrómetro. Puesto que los parámetros que miden varían en función del tiempo, todos los equipos descritos cuentan con la capacidad de almacenamiento y transmisión de datos a una computadora personal a intervalos programables. Además, su diseño permite soportar las condiciones del trabajo de campo. Como resultado de las pruebas de laboratorio en el pluviómetro, su error relativo máximo es del $4 \%$ para un rango de precipitación diaria menor a $500 \mathrm{~mm} / \mathrm{d}$. Por otra parte, el método de medición del evaporímetro redujo un factor de 5 el error relativo del sensor de presión empleado para medir el nivel del agua en un tanque de evaporación, resultando en una precisión del 98.5\%. Una característica singular de los dos dispositivos mencionados es que tienen la capacidad de auto calibrarse después de cada ciclo de trabajo. A su vez, el limnígrafo es capaz de medir el nivel de una corriente superficial con una precisión de hasta $58 \mu \mathrm{m}$, debido su sistema de posicionamiento electromecánico. En lo que respecta al freatímetro, éste mostró un error de medición respecto a su escala completa del 1.5\%. Como una ventaja adicional, este dispositivo mide el nivel freático a través de la determinación de la presión diferencial en lugar de la absoluta, eliminando con ello la necesitad de un equipo adicional para compensar el efecto de la presión atmosférica. Una vez calibrados los bloques de yeso empleados en el higrómetro, fue posible la determinación de la humedad relativa con una variación del $2.8 \%$ en el valor de resistencia eléctrica para un rango de temperatura entre $8^{\circ} \mathrm{C}$ y $17^{\circ} \mathrm{C}$. Para determinar los posibles efectos de desgaste en los componentes, las pruebas de campo tuvieron una duración de seis meses. Tras una serie de modificaciones en los diseños, fue posible construir los equipos de tal modo que no requirieron ningún mantenimiento una vez instalados. De los cinco dispositivos, el que exhibe una mayor exactitud es el limnígrafo, con resultados prácticamente iguales en el campo respecto de los obtenidos en el laboratorio. Como resultado de la optimización de los diseños, el costo de la fabricación de los cinco dispositivos fue de 1250 dólares, alrededor del 12\% del costo de equipos similares.

Palabras clave: instrumentación, pluviómetro, evaporímetro, limnígrafo, freatímetro, higrómetro.

\begin{abstract}
Knowledge of the water cycle is an essential requirement to evaluate, plan and develop water resources. In this sense, this paper describes the principles of equipment operation, design, results of laboratory tests and the installation in the field of five devices: a rain gauge, an evaporimeter, a limnometer, a water table meter and a hygrometer. As the parameters measured vary with time, all the devices described are capable of storing and transmitting the data to a personal computer at programmable intervals. Moreover, the
\end{abstract}


design enables the equipment to withstand field conditions. As a result of the laboratory tests on the rain gauge, its maximum relative error is $4 \%$ for a daily rainfall of less than $500 \mathrm{~mm} /$ day. In addition, the method of evaporimeter measurement reduced by a factor of 5 the relative error in the pressure sensor used to measure the water level in an evaporation pan, resulting in a precision of $98.5 \%$. A singular feature of both devices is the capability to self-calibrate after each duty cycle. Furthermore, the limnometer can measure the level of surface flow with a precision of $58 \mu \mathrm{m}$ by the use of electromechanical positioning. In the case of the water table meter, it shows a full scale measurement error of 1.5\%. As an additional advantage, this device measures the water table by determining differential instead of absolute pressure, thereby eliminating the need of additional equipment to compensate for the effect of atmospheric pressure. Once the gypsum blocks used by the hygrometer are calibrated, it is possible to determine the relative humidity with a variation of $2.8 \%$ in the electrical resistance for a temperature range between $8^{\circ} \mathrm{C}$ and $17^{\circ} \mathrm{C}$. In order to determine the possible effects of deterioration of the components, the field test took six months. After a series of design modifications, it was possible to construct the equipment in such a way that no corrective maintenance is required once it is installed. The most accurate of the five devices was the limnometer, which exhibited practically equal results in the laboratory and field. As a result of the design optimization, the manufacturing cost of the five devices was 1250 dollars, which is about $12 \%$ of the cost of similar equipment.

Keywords: instrumentation, rain gauge, evaporimeter, limnometer, water table meter, hygrometer.

\section{Introducción}

El agua es uno de los recursos naturales indispensables para el desarrollo de los seres vivos y, sin embargo, tanto su cantidad como su calidad actualmente se ven seriamente amenazadas. Si bien es cierto que se tienen bases de datos mundiales de variables meteorológicas, tales como precipitación y temperatura, a menudo existen problemas hidrológicos en los que se requiere el monitoreo puntual de ciertos parámetros a una menor escala espacial y con una mayor frecuencia.

Las investigaciones actuales en materia de hidrología tienden a estudiar zonas y a plantear métodos aplicables a escalas cada vez mayores, en contraste con los estudios puntuales comunes en el pasado. Además, es usual encontrar parámetros cuya determinación no presenta dificultades técnicas como la estimación de la precipitación o de la temperatura ambiental. Sin embargo, la aplicación de modelos matemáticos a menudo se ve limitada por la cantidad de parámetros que técnicamente es posible cuantificar (Misstear et al., 2009; Smerdon et al., 2009; Wang et al., 2009). Por consiguiente, el conjunto de dispositivos empleados en estudios geohidrológicos y su distribución espacial está en función de los objetivos de la investigación, la escala de estudio y los métodos de medición, de manera que se pueden encontrar desde sensores de temperatura en un solo pozo para medir el flujo subterráneo (Guaraglia et al., 2009) hasta redes de dispositivos para realizar balances hídricos u otras determinaciones (Wendland et al., 2007).

Bajo tales circunstancias, algunas investigaciones a menudo están limitadas por el costo del instrumental científico necesario para la toma de datos, aunado a los correspondientes gastos de envío e importación. Además, en el caso de requerir un equipo de fabricación extranjera, generalmente se plantean dificultades adicionales como la carencia de personal especializado que pueda brindar asistencia para el uso, reparación e, incluso, modificación de los dispositivos. Por otra parte, uno de los problemas técnicos que presenta mayores dificultades para la caracterización hidrogeológica in situ de la zona no saturada radica en la estimación de parámetros clave como el contenido de humedad, el potencial de presión, así como valores de concentración de soluto de una pluma de contaminación, entre otros (Hancock y Raine, 2001; Gee et al., 2003; Lapen et al., 2004; Fang y He, 2008), cuya determinación es particularmente sensible a pequeñas modificaciones ambientales, lo que supone el continuo mantenimiento de los dispositivos de medición.

Al margen de estos condicionantes, la International Association of Hydrological Sciences (Strangeways, 1984) en su momento llamó la atención sobre la relación que existe entre una mayor exactitud en las mediciones y el consecuente incremento en el costo de los equipos, especialmente en aquellos casos en los que hay una variabilidad espacial del parámetro a cuantificar; de suerte que resulta más útil emplear varios equipos con una exactitud menor que un solo equipo mucho más costoso. Por consiguiente, una forma de reducir los gastos es diseñar instrumentos que tengan la adecuada, pero no tan imprescindiblemente alta, exactitud (Strangeways, 1984).

En esta línea, el presente artículo muestra el desarrollo de cinco instrumentos de monitoreo de variables hidrológicas fabricados en México, por el momento no comercializados: un pluviómetro, un evaporímetro, un limnígrafo, un freatímetro y un higrómetro; todos ellos con la capacidad de contabilizar, almacenar y transferir los datos a una computadora personal. Por tanto, el objetivo del presente documento es describir los correspondientes diseños de modo que sus principios de funcionamiento sean lo más comprensibles posibles para su implementación en prácticamente cualquier país, debido a que sus componentes son de fácil adquisición. 


\section{Metodología}

La metodología utilizada en esta investigación se desarrolló en las siguientes cuatro etapas: 1) diseño y construcción de cada uno de los equipos, 2) realización de las pruebas de laboratorio, 3) pruebas de campo y 4) recopilación y transferencia de datos de los dispositivos, seguido del correspondiente análisis.

\subsection{Elementos comunes y principios de funcionamiento}

A pesar de que los dispositivos miden distintos parámetros, tienen varias características en común, ya que cuentan con un microprocesador PIC16F877A comercializado por la empresa Microchip. Así mismo, incluyen una memoria en la que se almacenan hasta 6,000 registros de las variables monitoreadas y una vía de comunicación para su transferencia posterior a cualquier computadora que posea un puerto paralelo; además del programa específico para la descarga de los datos, también desarrollado en el marco de esta investigación.

El principio básico de funcionamiento del pluviómetro, del evaporímetro y del freatímetro se basa en transformar la presión de la columna de agua en cuestión, medida a través de un sensor de presión, en una tensión eléctrica. Posteriormente se convierte a una señal digital para su almacenamiento y procesamiento. Por otra parte, el limnímetro consiste en un sensor que detecta la ausencia o presencia de agua, sujeto a un motor que acciona un sistema mecánico que actualiza la posición del sensor a través de un hilo de baja elasticidad. Finalmente, el higrómetro mide la resistencia eléctrica entre dos terminales conductoras contenidas en un bloque de yeso. A continuación se indican los puntos particulares de la metodología empleada para cada uno de los equipos.

\subsection{Pluviómetro y evaporímetro}

Las características más importantes consideradas para el diseño de este equipo son: emplear un método de medición en el que no se subestime el volumen de agua precipitada durante lluvias intensas, que los intervalos de almacenamiento sean programables, conservar los datos a pesar de una interrupción en el suministro eléctrico y ser capaz de medir la precipitación y la evaporación en una zona de estudio en la que no se congele el agua almacenada y cuya precipitación máxima diaria no exceda los $500 \mathrm{~mm} /$ día.

A fin de optimizar la cantidad de componentes, y con ello simplificar la construcción y las pruebas, se diseñó un solo sistema que mide tanto la evaporación como la precipitación. El elemento de medición principal de este dispositivo es un transductor de presión MPXV5004G de la empresa Freescale con un rango de medición de 0 a $4 \mathrm{kPa}$. A fin de disminuir el error en la medición de la evaporación, se emplea un método análogo al que se usa para medir la precipitación. En esta última, los pluviómetros generalmente tienen un área de captación mayor a la sección transversal del recipiente que contiene la lluvia, con la finalidad de colectar una mayor cantidad de agua, cuya altura pueda ser dividida entre la relación de ambas áreas, la de captura y la del recipiente. El resultado de esta técnica es disminuir el error por efecto de lectura de las mediciones.

En la Figura 1 se muestra un diagrama simplificado de la estructura del pluviómetro y de la del evaporímetro. Todos los recipientes de este equipo cuentan con sensores de ausencia o presencia de agua fijados en la parte inferior y superior, que indican a la unidad de procesamiento si están llenos o vacíos. La forma en la que se mide la precipitación consiste en verter mediante la bomba 1 (bl) el agua de lluvia colectada a intervalos programables del vaso $1(v 1)$ al vaso $2(v 2)$, en el que se ubica el transductor de presión, de modo que una vez concluida la medición se activa la bomba 2 (b2) para evacuar el agua del sistema. El caudal nominal de las bombas es de 2.5 litros por minuto, lo que es suficiente para trasvasar una cantidad equivalente a $760 \mathrm{~mm} / \mathrm{h}$ de lluvia, al considerar una superficie circular de captura de $25 \mathrm{~cm}$ de radio. Esta capacidad es más que suficiente para cuantificar las lluvias en México, cuya precipitación diaria máxima que resulta en inundaciones en algunos estados como Veracruz es del orden de 300 mm/día (Garnica y Alcántara, 2004).

Para medir el nivel del tanque de evaporación, cada veinticuatro horas se abre la válvula $v$ mostrada en la Figura 1.b, operación que permite el tiempo suficiente para que ambos niveles se igualen. Cuando esto ocurre, se cierra la válvula $v$, después de lo cual se produce el transvase de agua de $v 3$ a $v 2$. El volumen interior de $v 3$ es cinco veces superior al de $v 2$ para disminuir el error en las mediciones. Por ejemplo, por cada milímetro que aumente el nivel en $v 3$, el nivel aumenta cinco milímetros en $v 2$, que contiene el transductor, con lo que el error debido a la medición del transductor disminuye por un factor de 5. Para llevar a cabo mediciones con un nivel superior a la quinta parte del nivel de $v 3$, se requiere el vaciado de $v 2$. En el microprocesador se almacena el número de veces que esto ocurre, así como el nivel final medido con el transductor. En la Figura 1.c se muestra una fotografía con los elementos correspondientes al diagrama descrito.

Las pruebas de laboratorio consistieron en verter hasta en 5 ocasiones en el área de captación del pluviómetro 10 distintos volúmenes para determinar el rango de medición del pluviómetro, así como su precisión. En lo que respecta a la calibración de las mediciones del tanque de evaporación, se operó siguiendo el mismo procedimiento que con el pluviómetro. El tiempo de apertura de la válvula se determinó inicialmente con base en el principio teórico de vasos comunicantes (Sotelo, 1994). Posteriormente, se ajustaron dichos tiempos con las observaciones producto de las repeticiones a distintos niveles.

En las Figuras 1.a y 1.d se muestran las fotografías de los equipos ya instalados en el campo. El punto de ubicación de los mismos debe tomar en cuenta la proximidad a la zona de estudio y la disponibilidad de suministro eléctrico, evitando 
(a)

\section{Precipitación}

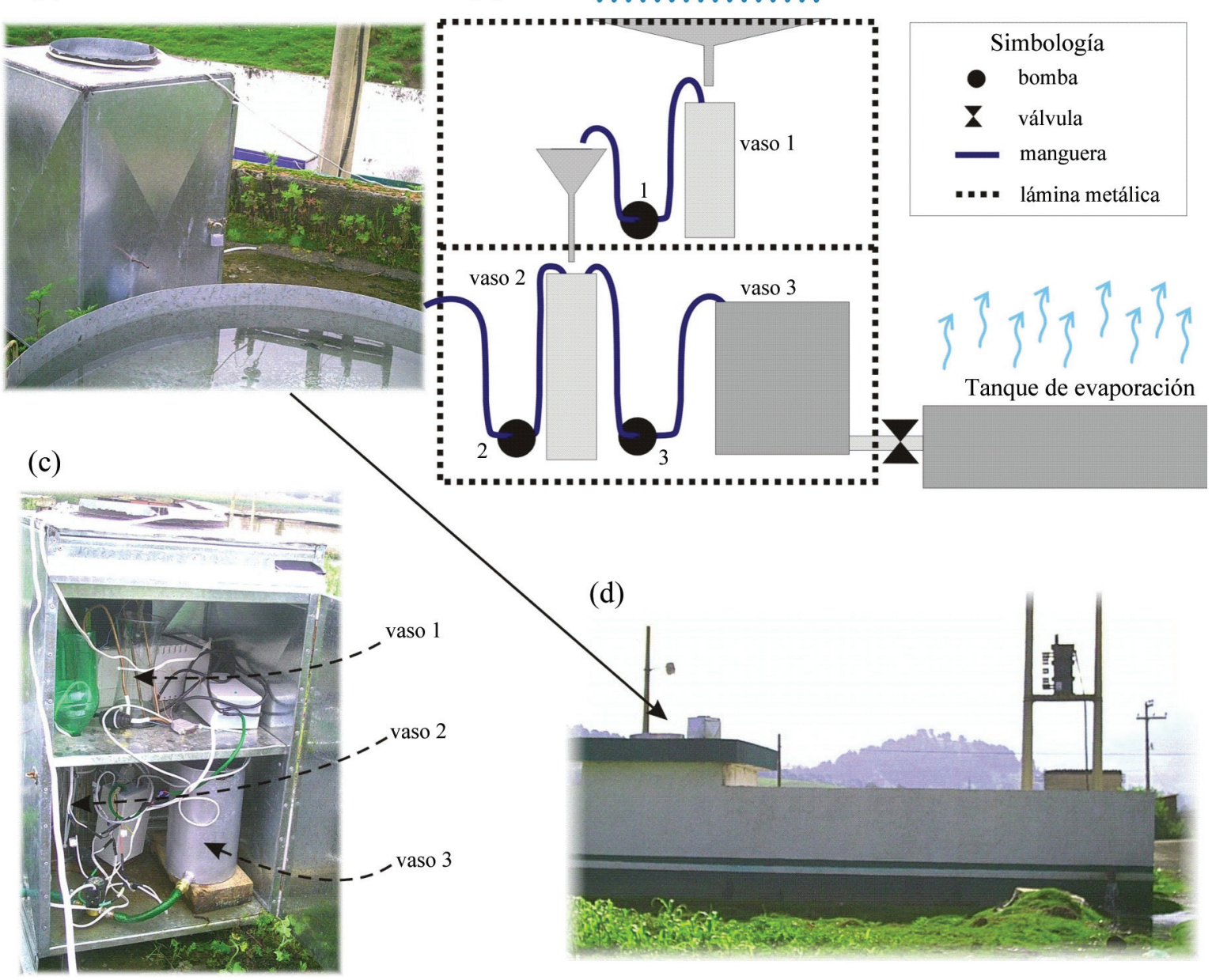

Figura 1. (a) Pluviómetro y evaporímetro; (b) diagrama simplificado de las partes que lo componen; (c) interior del pluviómetro; (d) instalación definitiva.

así costos adicionales de baterías o de celdas solares.

\subsection{Limnígrafo}

El objetivo de este dispositivo es determinar el caudal de una corriente superficial. Para lograrlo, el método empleado consiste en medir el nivel del espejo de agua de la corriente en cuestión, para que junto con una curva calibrada en función del caudal, se obtenga esta última variable.

La Figura 2 muestra un esquema simplificado del dispositivo, cuyo funcionamiento se fundamenta en que la flecha del motor de pasos mueve una transmisión a la que está unido un hilo del que pende un peso con dos terminales conductoras que le indican al microprocesador la ausencia o presencia de agua. La combinación del motor a pasos y una transmisión mecánica ofrece una gran precisión en el posicionamiento del peso que porta el sensor que detecta la presencia de agua.

El movimiento vertical del peso se realiza a intervalos de 15 minutos siguiendo el nivel de agua en la corriente superficial en cuestión, almacenándose a intervalos programables la posición de éste en la memoria del microprocesador como función del número de pulsos enviados al motor de pasos. La razón por la que se actualiza la posición a intervalos de 15 minutos es para impedir que el sensor quede sumergido en el agua y sea arrastrado por la corriente cuando el caudal se incremente.

Las pruebas de laboratorio, análogamente al procedimiento de calibración de los equipos antes descritos, consistieron en 5 determinaciones sucesivas efectuadas a 10 diferentes niveles de la lámina de agua.

Este dispositivo se instaló para su prueba en campo sobre el centro de un canal, debajo de un puente que proporcionaba protección contra la lluvia y fuera del alcance de las personas (Figura 2.b). La estructura de soporte para medir el caudal en el canal donde se instaló tuvo que cumplir dos requisitos mínimos: por un lado tener la suficiente resistencia mecánica para sostener el sistema con su fuente 
(b)

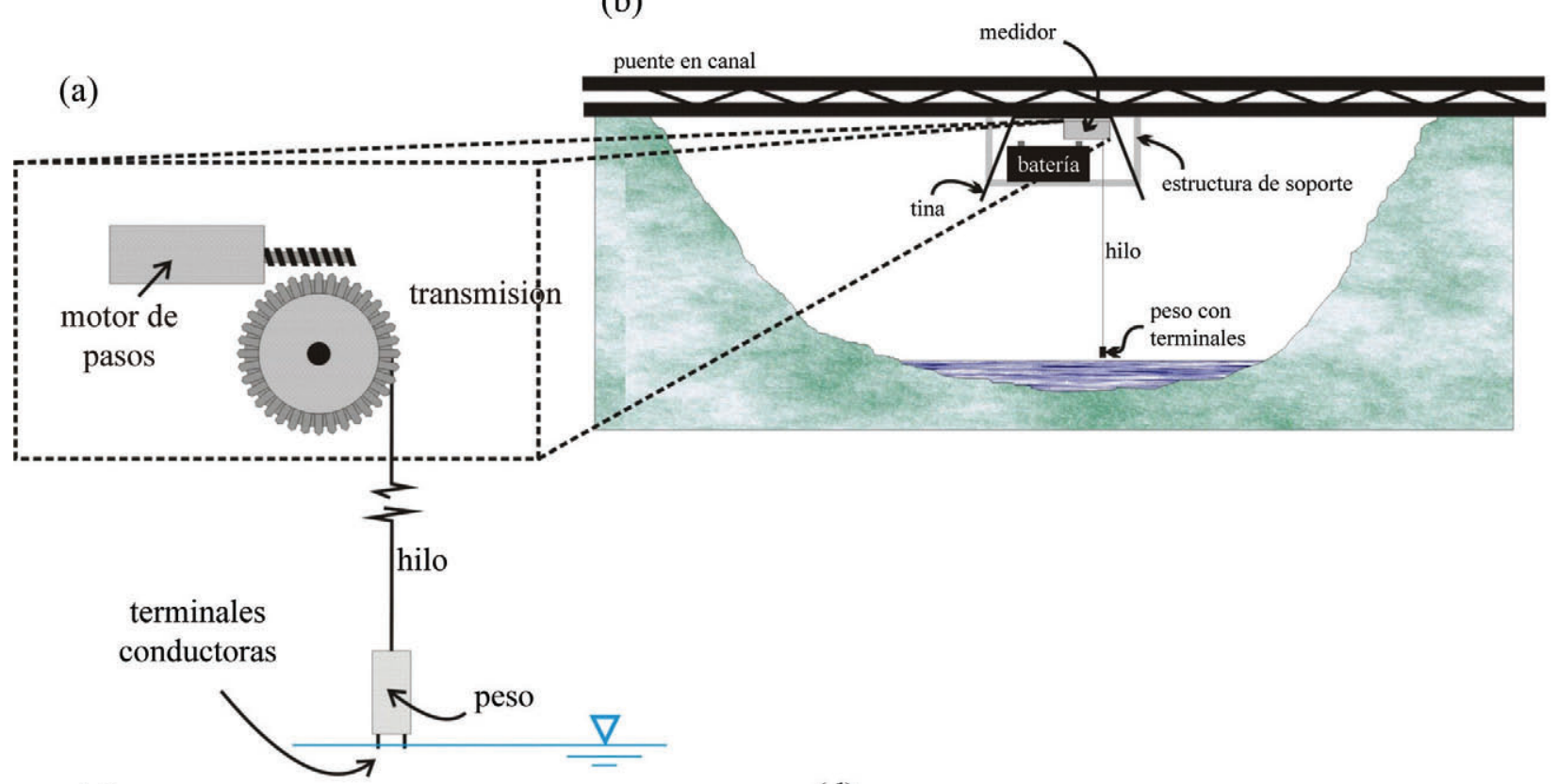

(c)

(d)

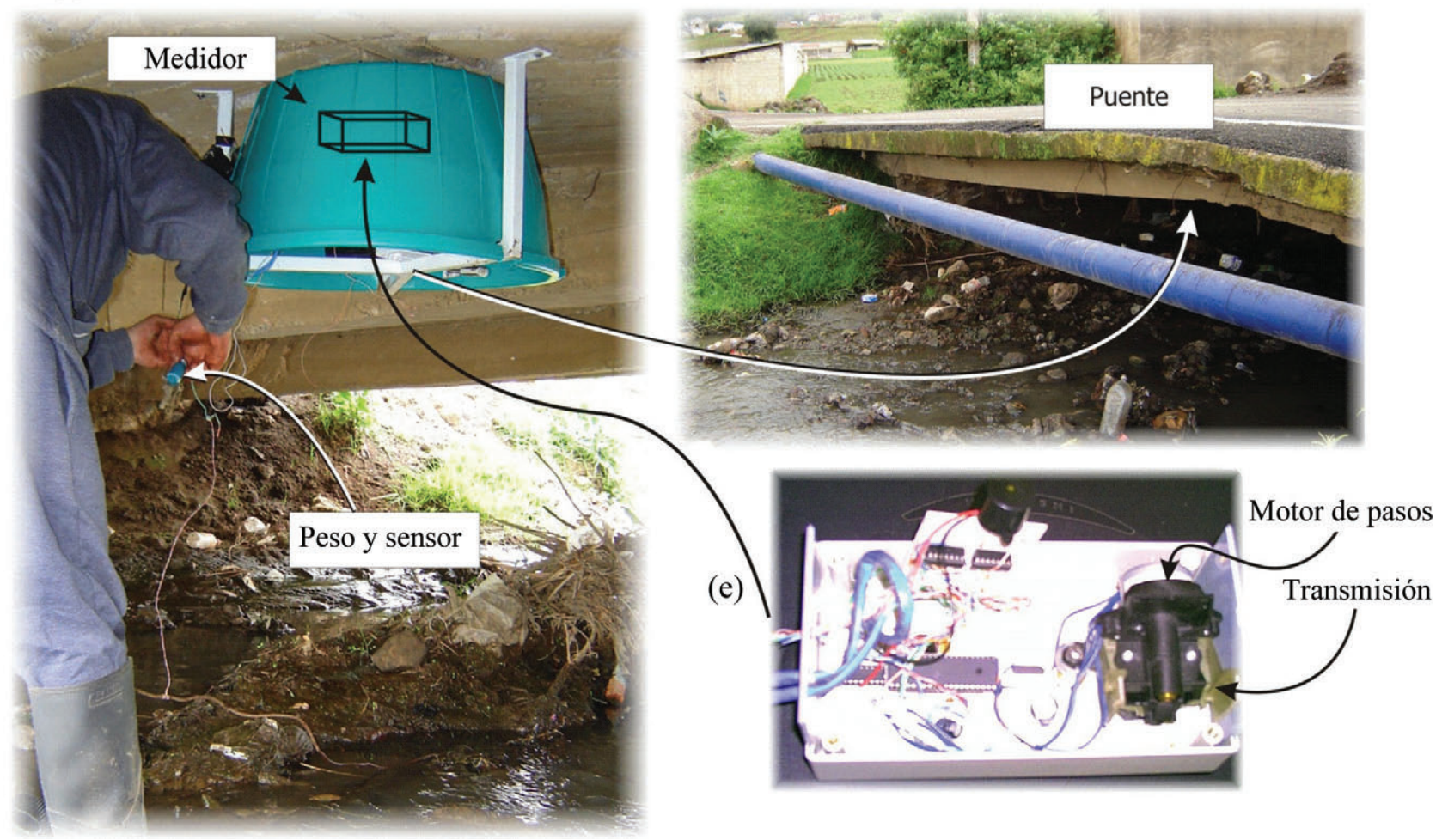

Figura 2. Limnímetro. (a) Esquema simplificado de estructura; (b) diagrama de instalación; (c) vista de la instalación; (d) fotografía de interior del sistema.

de alimentación, aun cuando el canal llegara a desbordarse $y$, por otro, que estuviera debidamente oculto para evitar que se produjeran averías o hurtos. Para evitar que el agua del canal dañase el medidor en situaciones de un eventual desbordamiento, tanto el dispositivo como su fuente de alimentación estuvieron protegidos por un contenedor con el lado descubierto hacia abajo, como se muestra en la Figura 2.b y 2.c. La presión del aire atrapado en su interior impide de manera natural que el equipo se moje a pesar que el canal esté a su máxima capacidad. 


\subsection{Freatímetro}

Uno de los parámetros fundamentales en la caracterización de la zona no saturada de cualquier acuífero es su espesor, el cual se puede determinar a partir de la medida de la profundidad del nivel freático en un pozo. Un método común que emplean los medidores comerciales es a través de la estimación de la presión ejercida por la columna de agua en cuestión. Sin embargo, los dispositivos que miden presión absoluta tienen el inconveniente de incluir el efecto de la presión atmosférica, por lo que se necesita un equipo adicional para restar dicha contribución (Rasmussen y Crawford, 1997). El método empleado en el freatímetro de este trabajo consiste en medir la presión diferencial entre la atmósfera y un tubo que se introduce en la columna de agua del pozo cuyo nivel se necesita calcular. La presión en el interior de dicho tubo es proporcional a la columna de agua. Puesto que la presión medida es diferencial, no necesita un medidor adicional para discriminar la presión atmosférica.

La estructura del freatímetro se muestra en la Figura 3. (a)

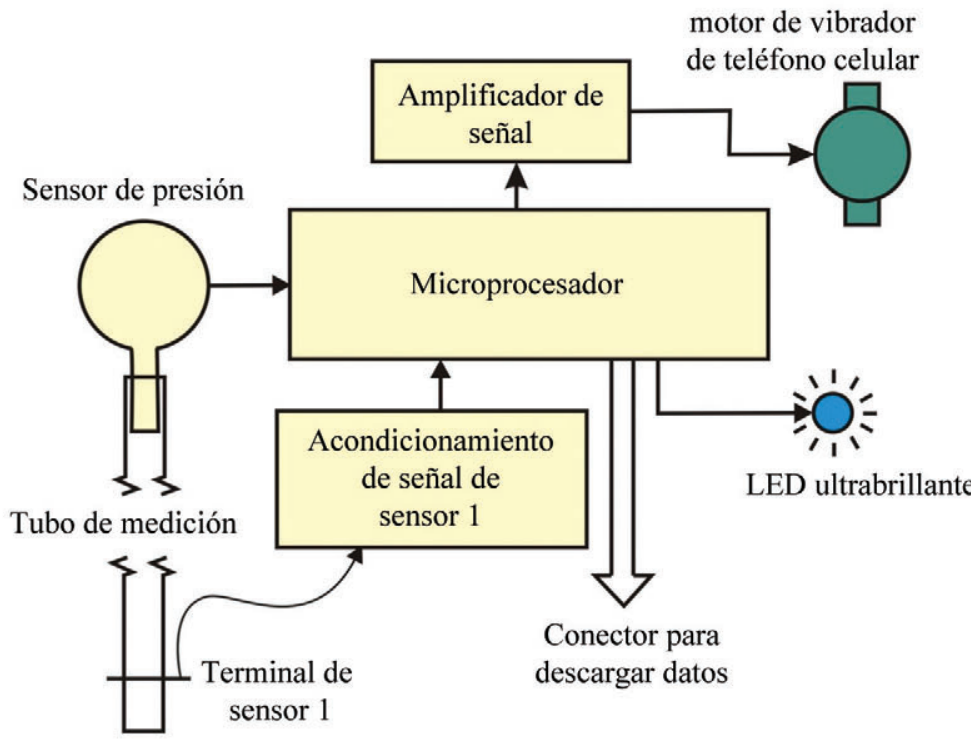

(b)

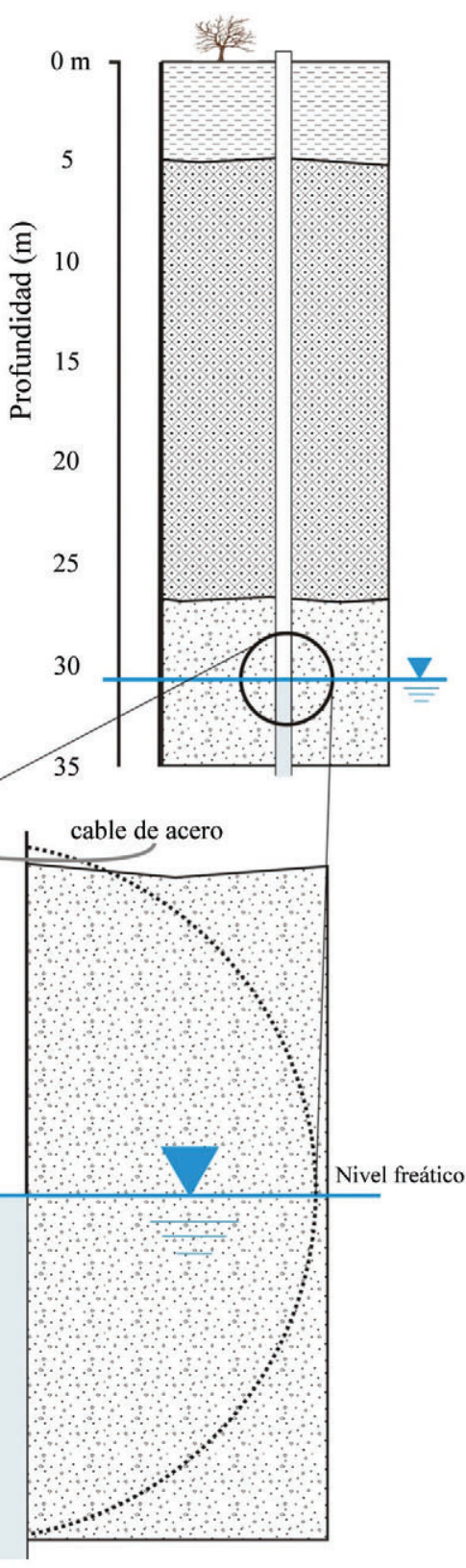
sensor 1

(c)

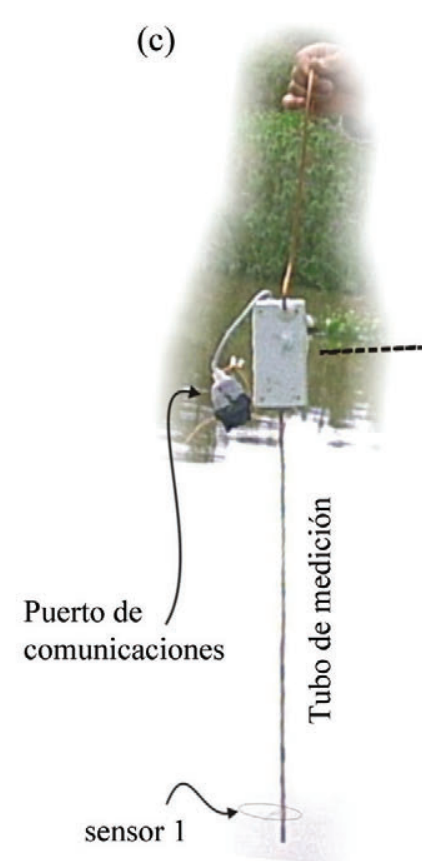

(d)

\section{d)}

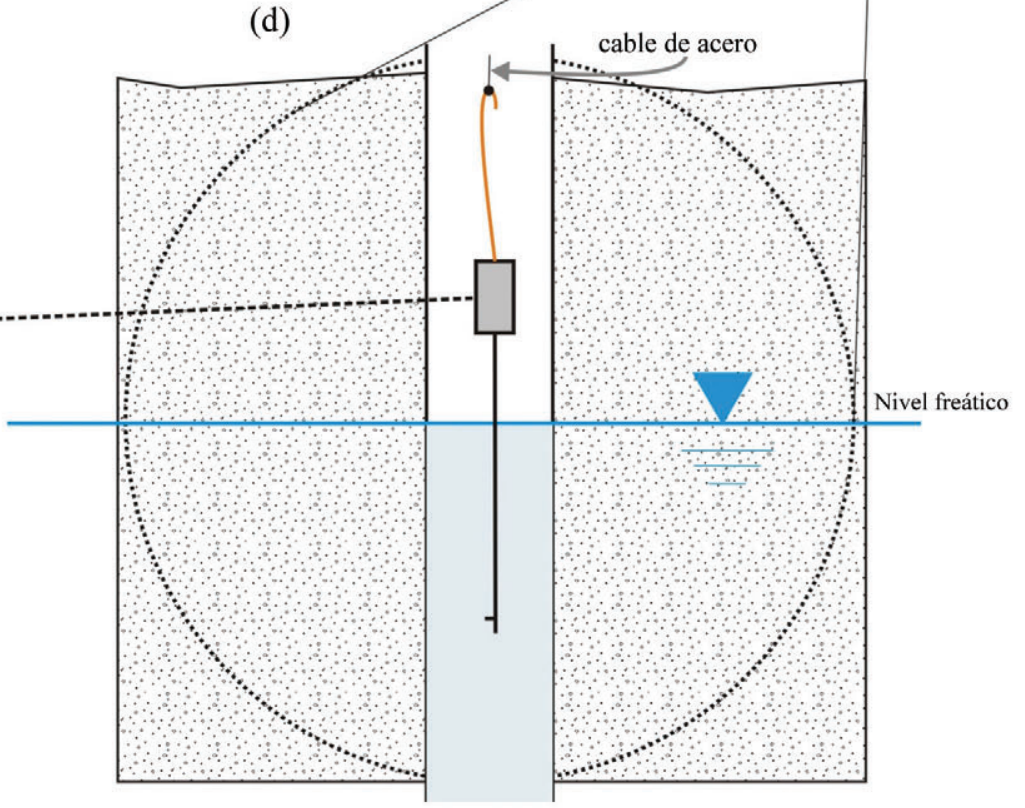

Figura 3. Freatímetro. (a) Esquema simplificado del dispositivo; (b) y (d) esquema de ubicación dentro de pozo; (c) fotografía del dispositivo. 
Este dispositivo incluye un transductor de presión diferencial cuya salida proporciona una tensión eléctrica proporcional al nivel. Dicho valor se acopla a un microprocesador que mide y almacena los valores. En ocasiones el nivel freático puede ser muy profundo, por lo que no siempre es posible visualizar con la suficiente seguridad cuando se alcanza el nivel freático. Por tal motivo, el equipo está dotado de dos medios alternativos para indicar que el sensor 1 , mostrado en la Figura 3.a y 3.c, ha alcanzado la superficie freática. El primer indicador consiste en la iluminación procedente de un $L E D$ (por sus siglas en inglés de Diodo Emisor de Luz), de tal intensidad que es posible visualizarlo a profundidades superiores a los cincuenta metros. Al mismo tiempo se activa durante cinco segundos un motor típico de los teléfonos portátiles para generar una vibración que se transmite a través del cable de acero, de manera mecánica, alertando al usuario a través del sentido de la vista y del tacto que el dispositivo ha detectado el nivel de agua. El grado de exactitud y sensibilidad del dispositivo se probó en el laboratorio siguiendo el mismo patrón de 5 medidas repetitivas de 10 diferentes niveles distribuidas en todo el rango de medición del instrumento.

En las Figuras 3.b y 3.d, se muestra la ubicación de su instalación dentro de un pozo. A pesar de que el rango de medición se limita a $40 \mathrm{~cm}$, puede ser útil en aplicaciones con una mayor variación ajustando la longitud del cable que sujeta el instrumento para actualizar su posición con respecto al nivel freático. Otra opción consiste en cambiar el transductor por otro de rango mayor, con el consecuente cambio en la longitud del tubo que se sumerge.

\subsection{Higrómetro}

Un método para determinar el contenido de humedad en el suelo consiste en la estimación de la conductividad eléctrica en bloques de yeso. Esta técnica, a pesar de ser considerada como cualitativa, permite aportar datos cuantitativos si se calibran las mediciones con los resultados obtenidos por otros procedimientos, entre los que destacan el empleo de sondas TDR (por las siglas en inglés de Reflectometría en el Dominio del Tiempo) y la técnica gravimétrica. Un problema potencial durante la medición es que al aplicar una corriente por un período prolongado (del orden de un segundo) se pueden producir gases en los electrodos, cambiando el valor de la resistencia aparente en mediciones sucesivas o con un tiempo de medición distinto (MEA, 1998).

Para evitar el problema mencionado, el equipo diseñado mide la resistencia eléctrica aplicando una corriente alterna durante 25 milisegundos por medición. Para esto, se emplea un circuito cuya frecuencia de oscilación está en función de un capacitor fijo y un resistor, cuyo valor es precisamente la resistencia eléctrica entre las terminales en el interior del bloque de yeso.

El microprocesador del equipo conecta de forma sucesiva hasta 4 bloques de yeso a través de un multiplexor analógico con el circuito oscilador. El microprocesador mide la frecuencia de los pulsos del oscilador, los cuales son directamente proporcionales al contenido de humedad, y almacena dichos valores a intervalos programables para su descarga posterior.

Para la calibración de los bloques de yeso se empleó la técnica gravimétrica. Consiste básicamente en tomar una muestra del suelo, pesarla, desecarla en horno a $110^{\circ} \mathrm{C}$ y volverla a pesar, de modo que la cantidad de agua extraída, determinada por diferencia de peso, es precisamente la contenida originalmente en la muestra (Sanders, 1998). Las condiciones físicas a las que se sometieron los bloques de yeso se basaron en el estudio realizado por Dela (2001), en donde se calibró la respuesta de cuatro de ellos, los cuales fueron manufacturados por la empresa Delmhorst Instruments Corporation. En esencia, el procedimiento de calibración consiste en evitar la histéresis de los bloques entre pruebas sucesivas, para lo cual cada bloque se sumerge en agua durante un tiempo suficientemente largo para que se sature por completo ( $8 \mathrm{~h}$ para dicho estudio). Partiendo de esa condición inicial, se mide la resistencia eléctrica de los bloques de yeso variando la temperatura o el contenido de humedad.

En los distintos apartados de la Figura 4 se puede apreciar el diagrama esquemático con los principales elementos que conforman el dispositivo, así como distintas fotografías correspondientes a su instalación en el campo. Para instalar el higrómetro se enterró éste junto con su fuente de alimentación a casi a dos metros de profundidad como se muestra en la Figura 4.a), mientras que los bloques de yeso se instalaron en dos grupos con dos bloques de yeso cada uno. El primer grupo se enterró a $50 \mathrm{~cm}$ y el segundo a casi $4 \mathrm{~m}$. Se realizó la instalación de ese modo para obtener mediciones a diferentes profundidades. Con el objetivo de que los bloques de yeso estuvieran en estrecho contacto con la matriz porosa, se empleó bentonita en una proporción de $30 \%$ con la tierra de cada perforación, aumentando de ese modo la velocidad con que se alcanza el estado estacionario, de acuerdo con las recomendaciones de Measurement Engineering Australia (MEA, 1998).

A fin de aislar y proteger el dispositivo del agua de infiltración, se diseñó una estructura que permite capturar y retener un volumen de aire en compresión, constituido por dos contenedores instalados como se muestra en la Figura 4.b, siguiendo el mismo principio de conservación de aire que el limnígrafo antes descrito. Igualmente, los cables que conectan los sensores al medidor también se enterraron, quedando totalmente invisible la ubicación del dispositivo, proporcionando así un entorno seguro para evitar el vandalismo y el daño por las inclemencias atmosféricas.

\section{Resultados}

En primer término se presentan los resultados de las pruebas de laboratorio efectuadas con cada dispositivo para 
(a)

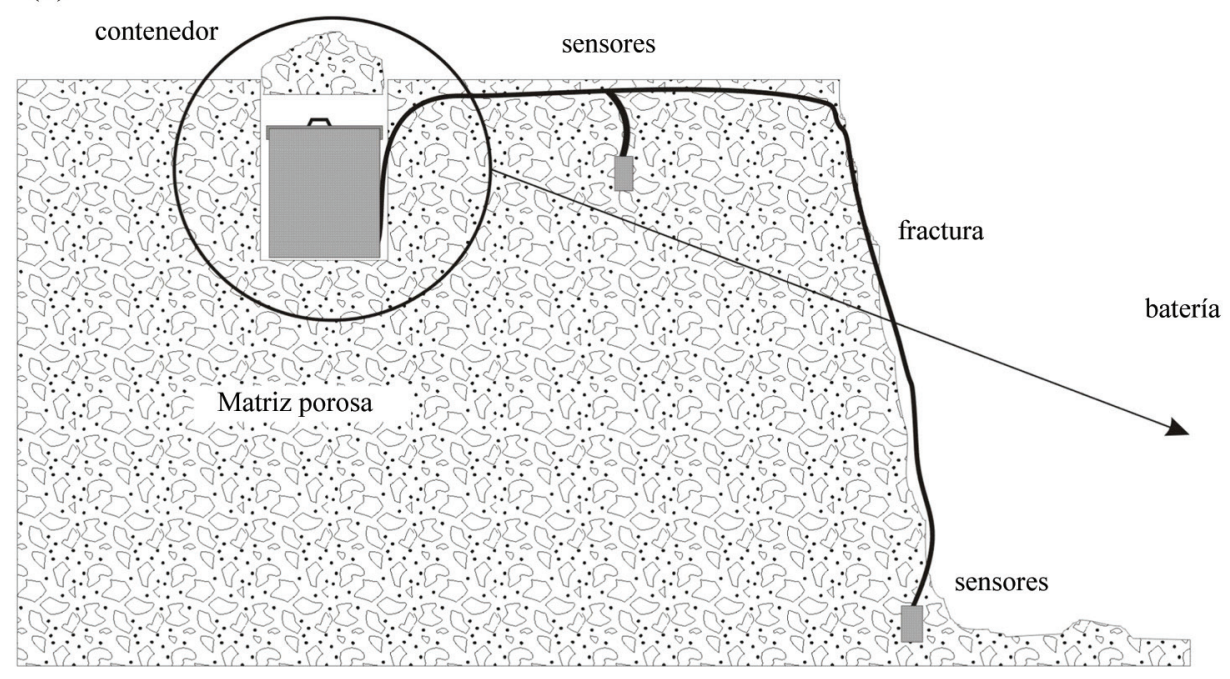

(b)
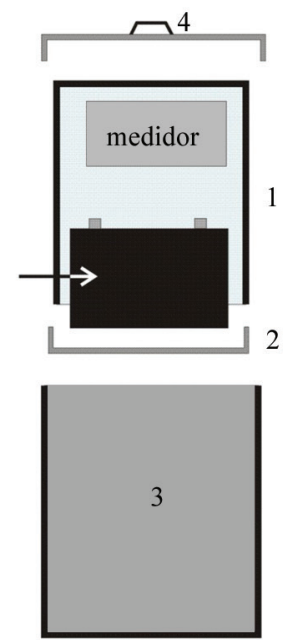

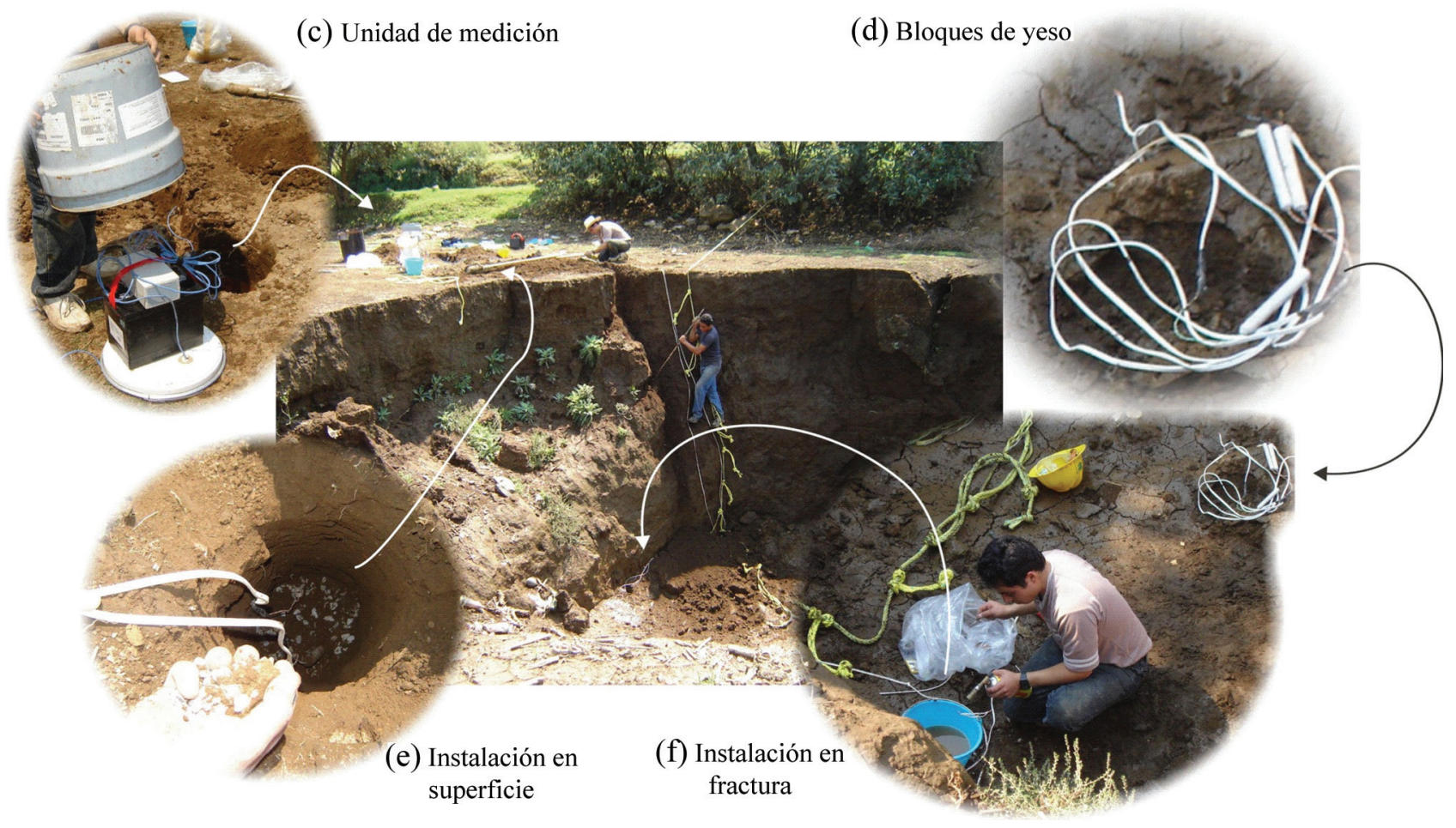

Figura 4. Higrómetro. (a) Esquema de instalación en el campo; (b) detalle de estructura de protección; (c) y (e) fotografías de instalación en superficie; (d) detalle de bloques de yeso; (f) instalación en fractura.

posteriormente mostrar los resultados obtenidos a partir de su instalación.

\subsection{Pluviómetro y evaporímetro}

Tanto en las Tablas 1 y 2 como en la Figura 5.a y 5.b se muestran los resultados de las pruebas de laboratorio realizadas con el pluviómetro y el evaporímetro. En los primeros volúmenes tabulados del pluviómetro (Tabla 1) se puede apreciar el error relativo de cada una de las cinco mediciones $(E R)$. Para determinar el peor escenario, se consigna en la decimocuarta columna el error relativo mayor 
Tabla 1. Volúmenes de agua medidos con el pluviómetro en pruebas de laboratorio y sus estadísticas.

\begin{tabular}{|c|c|c|c|c|c|c|c|c|c|c|c|c|c|c|}
\hline \multirow{2}{*}{$\begin{array}{l}\text { Vol. real } \\
(\mathrm{ml})\end{array}$} & \multicolumn{2}{|c|}{ Prueba 1} & \multicolumn{2}{|c|}{ Prueba 2} & \multicolumn{2}{|c|}{ Prueba 3} & \multicolumn{2}{|c|}{ Prueba 4} & \multicolumn{2}{|c|}{ Prueba 5} & \multicolumn{4}{|c|}{ Estadísticas } \\
\hline & Vol. (ml) & ER (\%) & Vol. (ml) & ER (\%) & Vol. (ml) & ER (\%) & Vol. (ml) & ER (\%) & Vol. (ml) & ER (\%) & $\begin{array}{c}\text { Pro-medio } \\
(\mathrm{ml})\end{array}$ & Desv. Est. & $\begin{array}{c}\text { ER mayor } \\
(\%)\end{array}$ & $\begin{array}{c}\text { ER mayor } \\
\text { EC }(\%)\end{array}$ \\
\hline 200 & 180 & 10 & 175 & 12.5 & 184 & 8 & 189 & 5.5 & 180 & 10 & 181.6 & 5.2 & 10 & 1 \\
\hline 400 & 398 & 0.5 & 358 & 10.5 & 347 & 13.3 & 419 & -4.8 & 372 & 7 & 378.8 & 29.5 & 7 & 1.4 \\
\hline 600 & 580 & 3.3 & 600 & 0 & 588 & 2 & 575 & 4.2 & 593 & 1.2 & 587.2 & 10 & 1.2 & 0.4 \\
\hline 800 & 831 & -3.9 & 811 & -1.4 & 811 & -1.4 & 837 & -4.6 & 753 & 5.9 & 808.6 & 33.2 & 5.9 & 2.4 \\
\hline 1000 & 994 & 0.6 & 989 & 1.1 & 1053 & -5.3 & 978 & 2.2 & 1015 & -1.5 & 1006 & 29.6 & -5.3 & -2.7 \\
\hline 1200 & 1203 & -0.3 & 1156 & 3.7 & 1205 & -0.4 & 1165 & 2.9 & 1190 & 0.8 & 1184 & 22.3 & 3.7 & 2.2 \\
\hline 1400 & 1425 & -1.8 & 1386 & 1 & 1384 & 1.1 & 1385 & 1.1 & 1380 & 1.4 & 1392 & 18.6 & -1.8 & -1.3 \\
\hline 1600 & 1599 & 0.1 & 1561 & 2.4 & 1580 & 1.3 & 1632 & -2 & 1593 & 0.4 & 1593 & 26.2 & 2.4 & 2 \\
\hline 1800 & 1776 & 1.3 & 1814 & -0.8 & 1808 & -0.4 & 1752 & 2.7 & 1721 & 4.4 & 1774 & 38.9 & 4.4 & 4 \\
\hline 2000 & 2009 & -0.5 & 1989 & 0.6 & 1996 & 0.2 & 1960 & 2 & 2052 & -2.6 & 2001 & 33.6 & -2.6 & -2.6 \\
\hline
\end{tabular}

Tabla 2. Niveles de agua medidos y sus estadísticas en pruebas de laboratorio de 4 de los dispositivos diseñados en este trabajo.

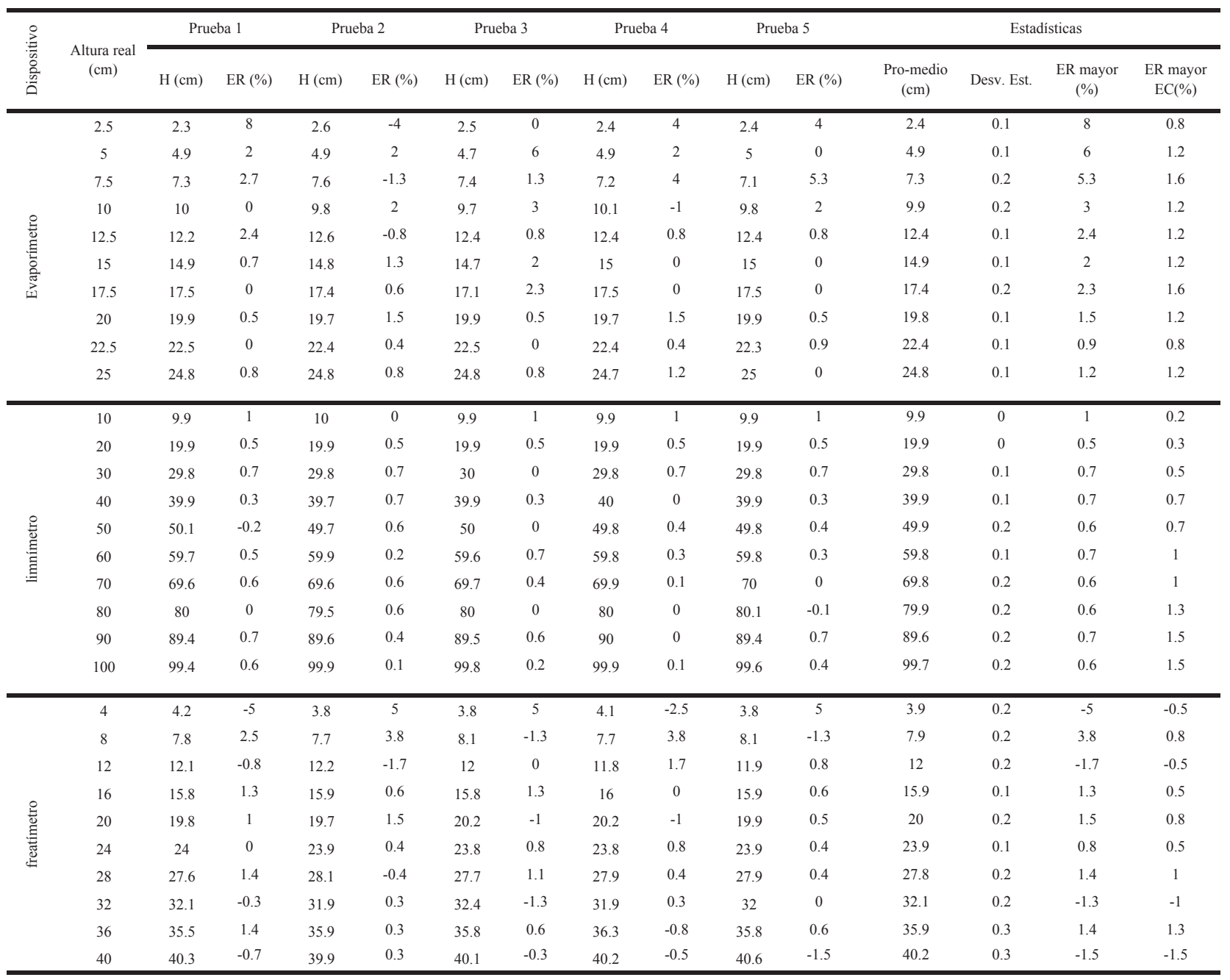

(ER mayor) de los cinco medidos, con un valor del 10\%, lo que supone una diferencia de $20 \mathrm{ml}$ respecto al volumen real de $200 \mathrm{ml}$. Si se compara dicho valor respecto al máximo medido 2,000 $\mathrm{ml}$, representa un error respecto a la escala completa (ER mayor EC) de tan solo el 1\%. El máximo error respecto a la escala completa es el correspondiente a 1,800 $\mathrm{ml}$, con un valor del $4 \%$, valor menor al que normalmente se tiene en muchos lugares producto de la heterogeneidad de la precipitación.

En la Figura 5.a se puede apreciar que el error en las 

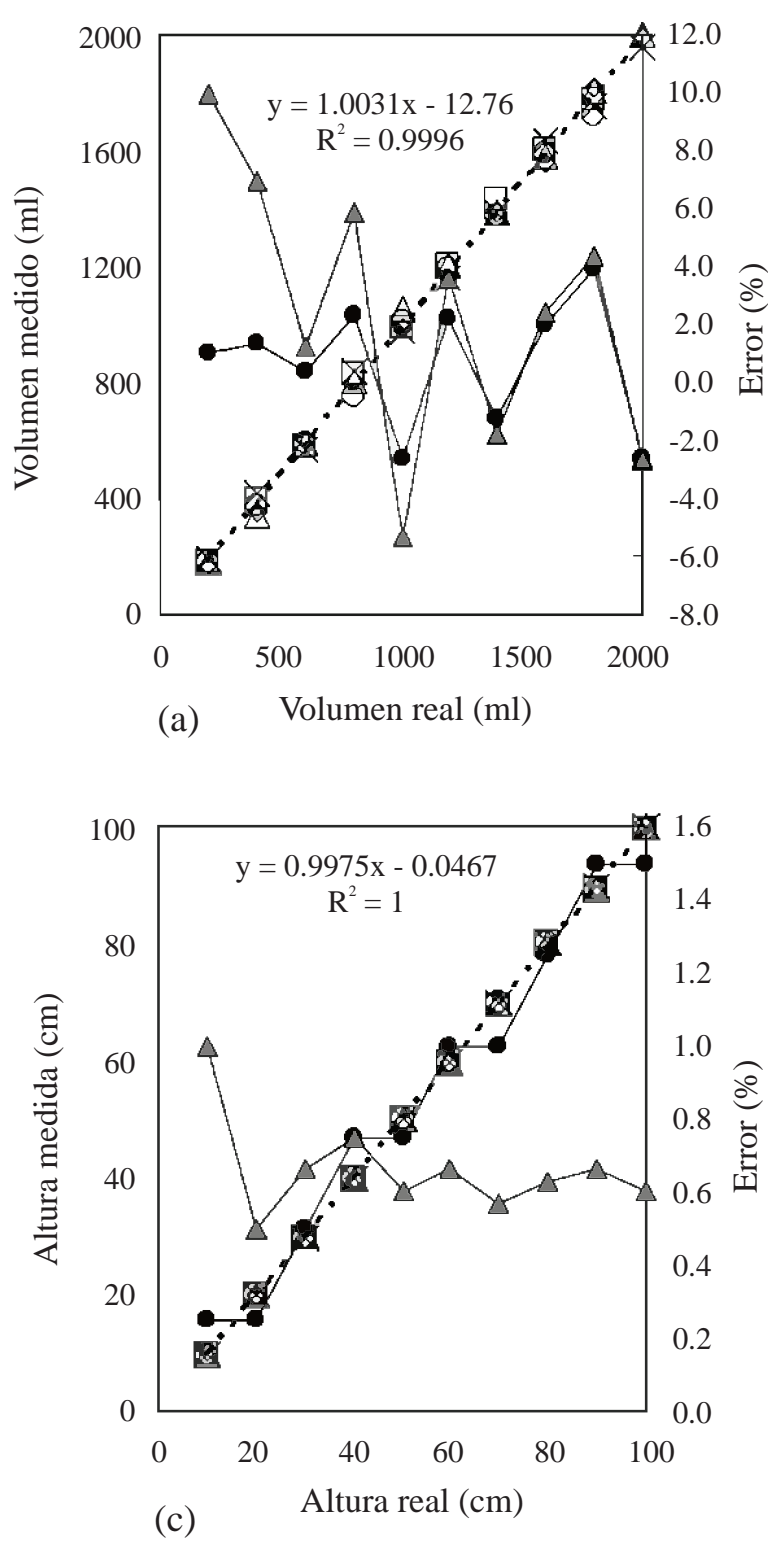
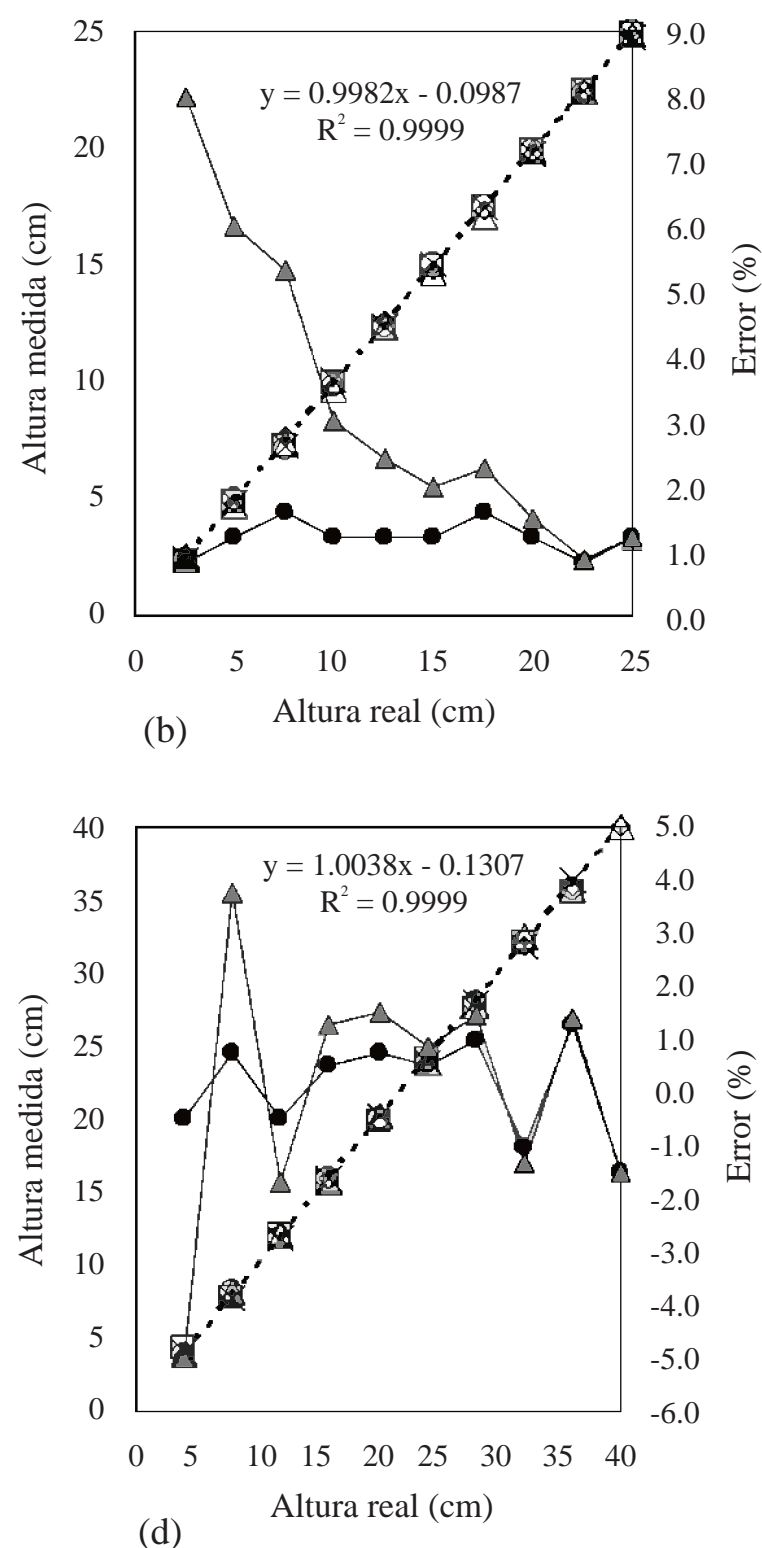

\begin{tabular}{|c|c|c|c|c|c|c|c|}
\hline$\square$ & Prueba 1 & $\diamond$ & Prueba 2 & $\triangle$ & Prueba 3 & $x$ & Prueba 4 \\
\hline 0 & Prueba 5 & - . - - & $\begin{array}{l}\text { Interpolación } \\
\text { lineal }\end{array}$ & $\rightarrow$ & $\begin{array}{l}\text { Error respecto a } \\
\text { escala completa }\end{array}$ & $\longrightarrow$ & Error relativo \\
\hline
\end{tabular}

Figura 5. Resultados de pruebas de laboratorio. (a) Pluviómetro; (b) evaporímetro; (c) limnígrafo y (d) freatímetro. Gráficos asociados a Tablas 1 y 2.

lecturas es mayor conforme la precipitación es menor, lo que en parte se debe al agua que queda retenida en el embudo de captación y en la tubería que comunica con el vaso $v 1$. Éste es un problema común documentado entre otros autores por Nystuen (1999) y Ward y Robinson (2000). También se puede apreciar en dicha figura que el coeficiente de determinación es cercano a la unidad $\left(\mathrm{R}^{2}=0.9996\right)$, producto de la alta linealidad del transductor de presión.

En lo que respecta al evaporímetro, en la Figura 5.b se observa una tendencia similar si se compara con los valores menores de nivel de agua. Sin embargo, es patente un error respecto a la escala completa de tan solo $1.6 \%$, mucho menor a la obtenida con la medición de la precipitación, lo que prueba la eficiencia del método descrito con anterioridad para disminuir el error en la medición de la evaporación, con un $\mathrm{R}^{2}=0.9999$. Además de que la magnitud del error es menor, la oscilación del error es incluso menor a la del pluviómetro, aun cuando los dos usan el mismo sensor para realizar las mediciones.

El consumo de energía de este equipo es de $1 \mathrm{~W}$ en 
reposo y de alrededor de $15 \mathrm{~W}$ en operación. Debido al tiempo de accionamiento de la válvula, de 35 minutos por cada medición de evaporación, y a los continuos accionamientos de las bombas, no se recomienda su uso con celdas solares. De ser necesaria su aplicación en zonas donde no hay cerca una red de suministro eléctrico, se podría emplear una fuente ininterrumpida de poder (UPS por sus siglas en inglés) con un acumulador de al menos 15 A-h para una operación de una semana.

Algunas de las ventajas de este sistema respecto a otros similares es que no contiene partes móviles que reduzcan la exactitud por el uso continuo del sistema. A pesar de que con el paso del tiempo las bombas pudieran tener un caudal menor, el sistema no requiere una calibración posterior debido a que el accionamiento de las mismas no está en función del caudal, sino del nivel determinado por terminales establecidas en distintos niveles.

Una de las limitaciones del equipo es su incapacidad para medir correctamente la evaporación cuando el agua está congelada; tampoco puede estimarse adecuadamente este parámetro cuando el tanque se desborda. En cuanto a la primera limitante, el período de mediciones no incluyó el invierno, por lo que no tuvieron lugar temperaturas tan bajas que congelaran el agua. En lo que respecta al segundo problema, hubo algunos días en los que efectivamente se desbordó el tanque, no obstante, para esos casos, la evaporación se consideró igual a la del día más cercano con el mismo régimen de lluvia. Futuros diseños pueden solucionar este problema mediante la apertura de una compuerta al superarse cierto nivel en el tanque de evaporación.

Para analizar sus diferencias y compararlas con la obtenida en el periodo estudiado, en la Figura 6.a se muestran las curvas de precipitación acumuladas de dos estaciones automáticas oficiales ubicadas en el valle de Toluca, las más cercanas a la zona donde se probaron los instrumentos. Se puede observar una diferencia entre la precipitación estimada respecto a la estación Nevado de Toluca de unos $30 \mathrm{~mm}$ a finales de agosto y una diferencia de unos $120 \mathrm{~mm}$ entre ambas estaciones oficiales. Lo anterior prueba que el rango de valores proporcionados por el equipo instalado en el campo concuerda con la realidad al ser menor la diferencia entre los valores estimados y los aportados por una estación oficial respecto de la que existe entre las dos estaciones oficiales. Además, es de interés reseñar las similitudes existentes entre los valores de precipitación circunscritos con los números 1 y 2 en la Figura 6.a. La diferencia entre los valores acumulativos también obedece a la carencia de datos para algunos días en las estaciones oficiales. El costo total del evaporímetro y el pluviómetro fue de alrededor de $\$ 380$ dólares estadounidenses (USD).

\subsection{Limnígrafo}

En conformidad con el diseño, el dispositivo construido no requirió ninguna modificación respecto a su planteamiento
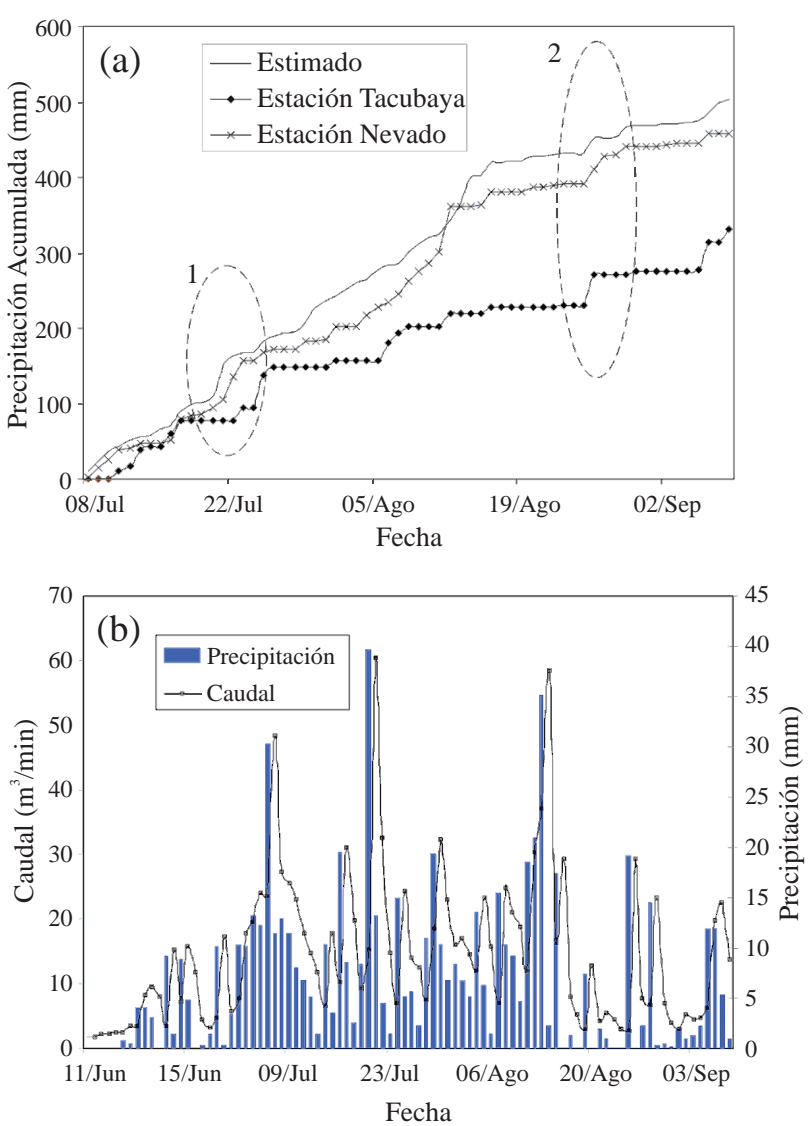

Figura 6. (a) Comparación de precipitación entre la zona donde se instaló el pluviómetro respecto a dos estaciones meteorológicas automatizadas cercanas a ésta. En (b) se compara la precipitación con el nivel medido en corriente de agua superficial.

inicial en lo concerniente a la metodología. Respecto a su respuesta, tuvo un comportamiento altamente lineal con un coeficiente de determinación de $\mathrm{R}^{2}=1$ para el rango empleado. Esto se debe a que el giro del motor es de $7^{\circ}$ por cada pulso y como la transmisión tiene una relación de 42 a 1, cada pulso genera un giro de $0.1666^{\circ}$ en el carrete que enrolla el hilo del que pende el sensor. Ahora bien, puesto que el carrete tiene $2 \mathrm{~cm}$ de diámetro, cada pulso del motor mueve el sensor que pende del sistema alrededor de $58 \mu \mathrm{m}$, esto hace que la variación mostrada en la Tabla 2 y la correspondiente Figura 5.c, obedezca más bien al enrrollamiento irregular del hilo en el carrete, que a una falla en el posicionamiento del sensor. Sin embargo, estas variaciones son aceptables y menores al $1.5 \%$ en todas las pruebas realizadas. De todos modos, si se reduce el ancho del carrete se mejora considerablemente el patrón de enrollamiento, pero la linealidad se vería afectada.

En la Figura 6.b se compara la precipitación con el caudal estimado en el canal de agua mediante el medidor descrito, constatándose la congruencia existente entre ambos conjuntos de datos.

A pesar de su alta precisión, el dispositivo presentó la desventaja de que cuando el canal transporta objetos como 
ramas o basura, éstos pueden adherirse al sensor que pende del sistema y romper el hilo que lo sujeta. Por otra parte, un factor que limita su utilización es que requiere una estructura de fijación sobre la parte central de la corriente superficial bajo análisis, además de la necesidad de elaborar una curva de gastos y su actualización eventual debido a la erosión del lecho del canal o del azolve, según sea el caso. El costo total del limnígrafo fue de aproximadamente \$290 (USD).

\subsection{Freatímetro}

En la última parte de la Tabla 2 se indica el desempeño del medidor de nivel freático con un error relativo del 5\% y un error respecto a su escala completa del 1.5\%. Además de su respectiva gráfica (Figura 5.d), se deduce que tiene un comportamiento altamente lineal con un coeficiente de determinación $\mathrm{R}^{2}=0.9999$.

En lo que respecta a su instalación, no fue necesario un acondicionamiento especial del punto seleccionado y únicamente en cada visita se actualizaba la posición del medidor cuando era necesario para que el nivel freático estuviera dentro del rango de medición del sistema de 0 a $40 \mathrm{~cm}$.

Las ventajas de este producto respecto a otros similares es su bajo costo, mayor exactitud y que mide presión diferencial, en contraste con los medidores de nivel comerciales que trabajan con presión absoluta, mismos que son susceptibles a cambios en la presión atmosférica y otros errores documentados por Rasmussen y Crawford (1997). Por su parte, entre sus desventajas cabe señalar su mayor tamaño (Figura 3.c), rango limitado y que no debe sumergirse en el agua. El costo total del freatímetro fue de \$280 (USD).

\subsection{Higrómetro}

En la Figura 7.a se presentan algunas de las curvas obtenidas en la calibración de los bloques de yeso. De 37 bloques fabricados y ensayados para ver cuáles tenían un comportamiento similar, tan solo 4 tuvieron una respuesta semejante. En la Figura 7.a se les identifica como seleccionados. También se ilustra el comportamiento de uno de los desechados como ejemplo. En las pruebas realizadas se observó un amplio rango de variabilidad en función del contenido de humedad, siendo el valor mínimo de resistencia eléctrica cercano a los $6,500 \Omega$ en condiciones de saturación (sumergidos en agua). En el otro extremo, al exponerlos a la intemperie, con una humedad atmosférica relativa en torno al $45 \%$ y $18^{\circ} \mathrm{C}$ de temperatura, alcanzaron el estado estacionario alrededor de las $7.5 \mathrm{~h}$ con un valor de resistencia en torno a $9,250 \Omega$, lo que implica un rango de escala completa de 2,750 $\Omega$. En la Figura 7.b se muestra la respuesta de los bloques seleccionados en función de la temperatura, constatándose para cada uno de ellos y para el rango entre $4^{\circ} \mathrm{C}$ y $18^{\circ} \mathrm{C}$ que existe un rango de variación aproximada de $30 \Omega$, lo que representa un $1.09 \%$ respecto a la escala completa, por lo que su variación respecto a la temperatura se puede considerar despreciable.

Al comparar las respuestas de los bloques de yeso seleccionados con los resultados procedentes del estudio de Dela (2001), en el que se describe en detalle la calibración de un conjunto de bloques de yeso comerciales, se observan diferencias significativas concernientes a dos principales ventajas que los primeros presentan respecto a los del citado estudio. La primera ventaja tiene que ver con la diferencia en la resistividad entre los distintos bloques, establecida en término medio en unos $100 \Omega$. La variación de resistividad de los bloques en el presente estudio es menor que la reportada para los bloques comerciales del anterior estudio, con un intervalo de unos $13 \mathrm{k} \Omega$ para $0^{\circ} \mathrm{C}$ y de $5 \mathrm{k} \Omega$ para $25^{\circ} \mathrm{C}$ (Dela, 2001).

La segunda ventaja consiste en que la variación relativa de la resistencia entre los diferentes bloques es casi independiente de la temperatura, existiendo únicamente errores de corrimiento, comúnmente conocidos como offset. Así, para los bloques comerciales el comportamiento tiene una tendencia lineal y la diferencia a temperaturas cercanas a $0^{\circ} \mathrm{C}$ es poco más de dos veces que a $25^{\circ} \mathrm{C}$, mientras que para los de esta investigación la diferencia mayor se mantiene aproximadamente constante e igual a $100 \Omega$ para el mismo rango de temperatura.

Por otra parte, el consumo de energía del equipo resultó ser muy bajo, de tan solo $5 \mathrm{~mA}$ en reposo y 20 $\mathrm{mA}$ en operación, no precisándose reemplazar ni una sola vez la batería durante los seis meses que duró la prueba en el campo. Además, es de interés señalar que la unidad de medida permaneció seca aun cuando la zona estuvo parcialmente inundada durante algunos días, por lo que la estructura de protección, necesaria para la instalación en particular, funcionó como se esperaba. El costo total del higrómetro fue de $\$ 303$ (USD).

\section{Conclusiones}

En respuesta a la necesidad de establecer un equilibrio entre el costo y la exactitud de equipos capaces de cuantificar variables hidrológicas, en el presente artículo se describe el principio de funcionamiento, diseño, construcción e instalación de cinco dispositivos, con un rango de error en las mediciones inferior al 5\% respecto a la escala completa de cada uno de ellos. Los parámetros cuantificados por dichos dispositivos fueron: precipitación, evaporación, el nivel de la lámina de agua en una corriente superficial, el nivel de la superficie freática y el contenido de humedad del suelo.

El grado de validez de los datos aportados por el pluviómetro se puso de manifiesto tanto en pruebas de laboratorio como en el campo. En el primer caso los ensayos efectuados indicaron un error relativo máximo del $4 \%$ respecto a la escala total del instrumento. En el segundo, la validación consistió en comparar la información 

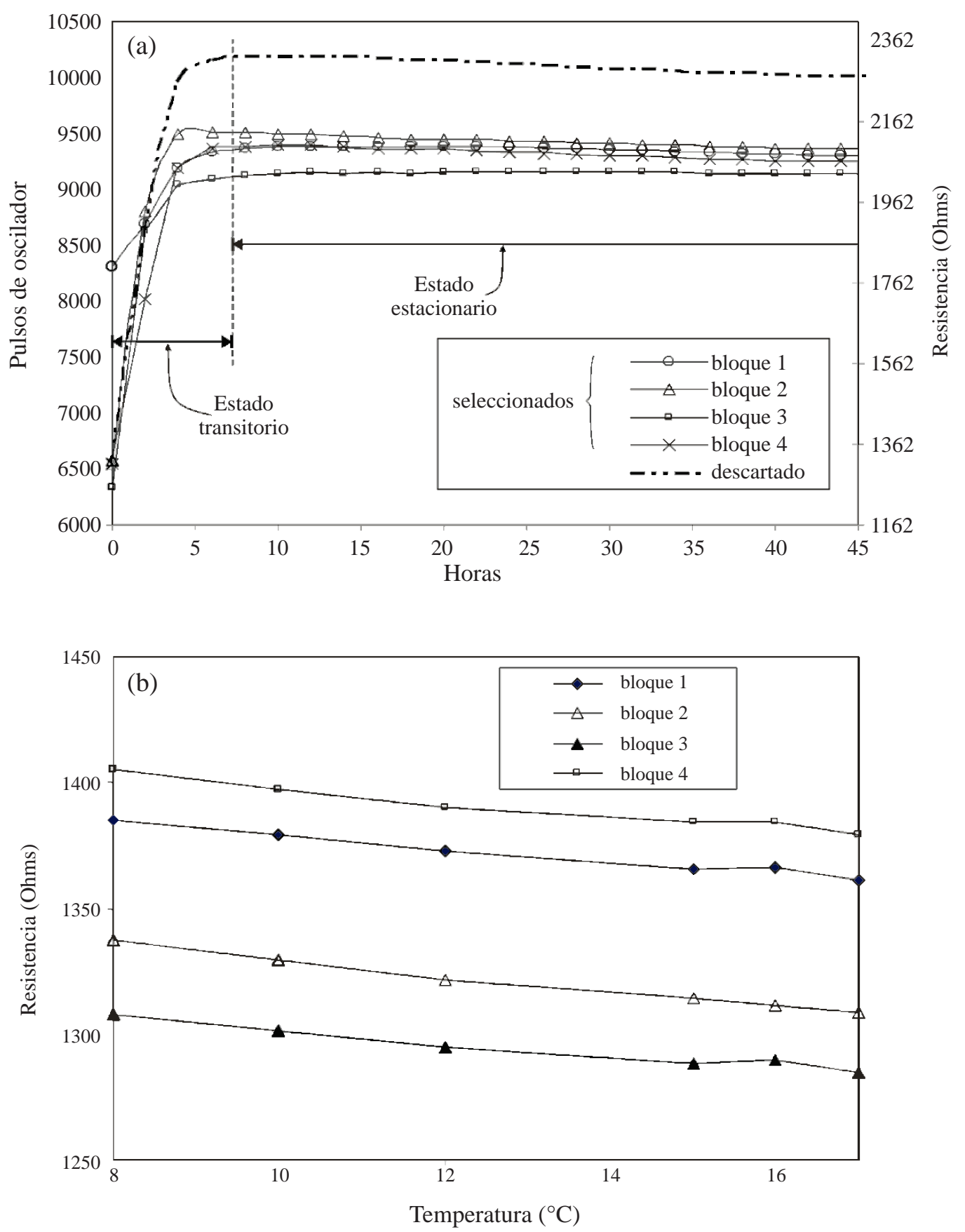

Figura 7. Resultados de calibración del higrómetro. (a) Curvas empleadas para la selección y calibración de los bloques de yeso a $18{ }^{\circ} \mathrm{C}$ y $45 \%$ de humedad atmosférica relativa; (b) respuesta de los cuatro bloques seleccionados en función de la temperatura.

contabilizada por el dispositivo durante el periodo de estudio con la de dos estaciones meteorológicas cercanas, obteniéndose una precipitación acumulada de casi 500 $\mathrm{mm}$, frente a los $458 \mathrm{~mm}$ y $330 \mathrm{~mm}$ de las otras estaciones, cuya diferencia se puede atribuir a la heterogeneidad de la distribución pluvial y a la falta de datos de precipitación entre las estaciones. Los valores de evaporación obtenidos se encuentran dentro de los límites convencionales descritos en la literatura hidrometeorológica.

Las pruebas realizadas con el limnígrafo pusieron de manifiesto la existencia de una linealidad tal que alcanzó un coeficiente de determinación $\mathrm{R}^{2}=1$, el mayor de todos los dispositivos diseñados, con tan solo un $1.5 \%$ de error respecto a la escala completa del aparato, que se debe al irregular modo como se enrolla el hilo del que pende el sensor que detecta la presencia de agua. Además, la 
información generada en el trabajo de campo puso en evidencia un alto grado de congruencia con los datos obtenidos con el pluviómetro, lo que sirve como una comprobación adicional de ambos dispositivos.

Por su parte, el medidor del nivel freático mostró, igualmente, un error relativo del $1.5 \%$, aunque con un coeficiente de determinación en las pruebas de laboratorio ligeramente menor: $\mathrm{R}^{2}=0.9999$. En las verificaciones que se hicieron en el campo coincidiendo con visitas periódicas, al objeto de hacer un seguimiento de las distintas instalaciones y efectuar el volcado de la información, no mostró deterioro alguno durante su utilización en el periodo estudiado. Al comparar los apartados a, b y d de la Figura 5, se percibe la diferencia de exactitud entre los tres aparatos que usan el mismo tipo de sensor, evidenciándose que el instrumento que mide la evaporación es más exacto, lo que resulta necesario dada la naturaleza misma de esta variable que cambia más lentamente que la precipitación, aunque de manera más continua.

Una parte decisiva en el desarrollo del higrómetro supuso el establecimiento de las características precisas de los bloques de yeso. En este sentido, y si bien es cierto que se tuvieron que desechar más de 30 unidades que no guardaban similitud, en términos de su respuesta al ciclo de sumergirlos en agua tras su exposición a la intemperie, cuatro de ellos mostraron una diferencia máxima de 100 $\Omega$ en un rango de $2,750 \Omega$, lo que represente un $3.63 \%$. Así mismo, mostraron una ligera variación con respecto a la temperatura de tan solo el $1.09 \%$ respecto a la escala completa para un rango de $8^{\circ} \mathrm{C}$ a $17^{\circ} \mathrm{C}$.

El diseño de los equipos ha mostrado un elevado desempeño en términos de funcionalidad y costo. En concreto, el valor de los elementos necesarios para su fabricación fue de poco más de $\$ 1,250$ (USD). Esto constituye alrededor de la octava parte de lo que hubiesen supuesto la adquisición y los gastos de importación de equipos similares; aunque dicha fracción puede variar en función del fabricante, distribuidor y país. Además, los costos de operación y mantenimiento disminuyen radicalmente por ser de fabricación nacional. Para el conjunto de dispositivos presentado no fue necesario realizar ningún gasto de mantenimiento una vez terminadas las pruebas de laboratorio.

Finalmente, dado que la heterogeneidad de los medios geológicos y de los fenómenos meteorológicos a menudo superan el error máximo, de $5 \%$, alcanzado con los dispositivos descritos, parece más que evidente que en zonas de estudio extensas resultaría más útil contar con varios equipos que midan parámetros con una precisión aceptable que un menor número de ellos con un elevado grado de exactitud. Los dispositivos presentados cumplen ambos requerimientos, a saber, el necesario bajo costo que permite la fabricación de varios de ellos, junto a una exactitud superior a la de la heterogeneidad existente, por lo general, en el medio natural.

\section{Agradecimientos}

Los autores agradecen por el financiamiento de este proyecto al Consejo Nacional de Ciencia y Tecnología (CONACyT), a la Comisión Geológica del Canadá, Recursos Naturales Canadá, División Québec (CGCQuébec/INRS), a la Universidad Autónoma del Estado de México (proyecto UAEM-2229) y al Departamento de Geodinámica de la Universidad del País Vasco. También por las facilidades otorgadas por el Ministère des Relations Internationales de Québec, Canadá y a la Dirección de Política y Cooperación Internacional en Ciencia y Tecnología del CONACyT, así como a la Comisión Nacional del Agua (CONAGUA) por hacer disponible la información que tiene a su cargo. Además reconocemos los valiosos comentarios y sugerencias de los Dres. Jordi Piña Iglesias, José Bolzicco y Aline Concha Dimas que mejoraron el contenido del artículo.

\section{Referencias}

Dela, B.F., 2001, Measurement of soil moisture using gypsum blocks, Hørsholm, Dinamarca, By og Byg-Danish Building and Urban Research, By og Byg Documentation 004, 28p.

Fang, H., He, Y., 2008, A Pocket PC based field information fast collection system: Computers and Electronics in Agriculture, 61, 254-260.

Garnica, R.J., Alcántara, I., 2004, Riesgos por inundación asociados a eventos de precipitación extraordinaria en el curso bajo del río Tecolutla, Veracruz: Investigaciones Geográficas, 55, 23-45.

Gee, G.W., Ward, A.L., Sisson, J.B., Hubbell, J.M., Myers, D.A., Sydnor, H.A., 2003, Hydrogeology Characterization Using Vadose Zone Monitoring Tools: Status Report: Richland, Washington, Pacific Northwest National Laboratory, Reporte Técnico PNNL-14115, 63 p.

Guaraglia, D.O., Pousa, J.L., Kruse, E.E, Mayosky, M.A., 2009, A Rotary Thermal Probe for Measuring Groundwater Velocity: Instrumentation Science and Technology, 37, 303 - 318.

Hancock, N., Raine,S., 2001, Advances in soil instrumentation: Computers and Electronics in Agriculture, 31, 211-212.

Lapen, D.R., Topp, G.C., Edwards, M.E., Gregorich, E.G., Curnoe, W.E., 2004, Combination cone penetration resistance/water content instrumentation to evaluate cone penetration-water content relationships in tillage research: Soil and Tillage Research, 79, $51-62$.

Measurement Engineering Australia (MEA), 1998, Resurrecting the Gypsum Block for Soil Moisture (en línea), Disponible en: http:// www.mea.com.au/files/publications/PUB-SM04.PDF, Fecha de consulta: 4 de febrero de 2009.

Misstear, B.D.R., Brown, L., Johnston, P. M., 2009, Estimation of groundwater recharge in a major sand and gravel aquifer in Ireland using multiple approaches: Hydrogeology Journal, 17: 693-706.

Nystuen, J.A., 1999, Relative performance of automatic rain gauges under different rainfall conditions: Journal of Atmospheric and Oceanic Technology, 16, 1025-1043.

Rasmussen, T.C., Crawford, L.A., 1997, Identifying and removing barometric pressure effects in confined and unconfined aquifers: Ground Water, 35, 502-511.

Sanders, L.L., 1998, A manual of field Hydrogeology: New Jersey, E.U.A., Prentice Hall, 381 p.

Smerdon, B.D., Allen, D.M., Grasby, S.E., Berg, M.A., 2009, An approach for predicting groundwater recharge in mountainous watersheds: Journal of Hydrology, 365, 156-172.

Sotelo, G., 1994, Hidráulica General, Tomo 1: Fundamentos: México, D. F., Limusa, 564 p. 
Strangeways, I.C., 1984, Low cost hydrological data collection, en Proceedings of the IAHS Symposium Challenges in African Hydrology and Water Resources: Harare, Zimbawe, IAHS Publication 144, 229-233.

Wang, T., Zlotnik, V.A., Simunek, J., Schaap, M.G., 2009, Using pedotransfer functions in vadose zone models for estimating groundwater recharge in semiarid regions: Water Resources Research, 45, W04412.

Ward R.C., Robinson M., 2000, Principles of Hydrology: Glasgow, Escocia, McGraw-Hill, 450p.
Wendland, E., Barreto, C., Gomes, L.H., 2007, Water balance in the Guarani Aquifer outcrop zone based on hydrogeologic monitoring: Journal of Hydrology, 342, 261-269.

Manuscrito recibido: Marzo 13, 2009.

Manuscrito corregido recibido: Agosto 20, 2009.

Manuscrito aceptado: Septiembre 15, 2009. 